\title{
Comparação dos resultados da aplicação de calor local e crioterapia no alongamento de isquiotibiais em adolescentes
}

\author{
Comparison of the results of local heat application and \\ criotherapy in the stretching of hamstring in adolescents
}

\author{
Nathália Fernandez de Castro, ${ }^{1}$ Marcelo Faria Silva, ${ }^{2}$ Daniel Carlos Garlipp' \\ 'Universidade Luterana do Brasil (ULBRA/RS), Canoas, RS, Brasil. \\ 2Centro Universitário Metodista (IPA/RS), Porto Alegre, RS, Brasil.
}

Recebido em: 28/12/2016 / Aceito em: 17/02/2017 / Publicado em: 31/03/2017

dcgarlipp@gmail.com

\section{RESUMO}

Objetivo: avaliar a eficácia de dois recursos térmicos no alongamento de isquiotibiais em adolescentes. Método: estudo clínico controlado não randomizado com 45 adolescentes do sexo masculino de 13 e 16 anos. Indivíduos com lesão musculoesquelética em membros inferiores, déficit de sensibilidade ou praticantes de atividade física que possibilitasse ganho da amplitude de movimento (ADM) foram excluídos do estudo. A amostra foi dividida em três grupos, sendo crioterapia, calor local e controle. Os grupos foram avaliados no mesmo dia, em horários diferentes. Com o auxílio de um goniômetro, utilizado antes e após a intervenção, foi solicitada uma flexão de quadril juntamente com uma extensão de joelho e dorsiflexão de tornozelo até o máximo de amplitude possível. Após a primeira medida, foram realizadas as intervenções com bolsas de gel, por 20 minutos, em temperatura abaixo de zero graus Celsius no grupo 1 , e calor no grupo 2, cuja temperatura era em média de $43^{\circ} \mathrm{C}$, diretamente sobre o ventre muscular. As temperaturas foram controladas com o auxílio de um termômetro. Após a intervenção, foi realizada nova medida. No grupo controle foram realizadas apenas duas medidas com o goniômetro espaçadas por 20 minutos. Para a estatística descritiva foram utilizadas a média e o desvio padrão. Para a comparação entre grupos foi utilizada a análise de variância de uma via com post-hoc de Tukey. Já para a análise dentro de cada grupo foi utilizado o teste $t$ pareado. Para a análise dentro de cada grupo foi utilizado o teste $t$ pareado. Resultados: os dois grupos experimentais (crioterapia e calor local) aumentaram efetivamente a $A D M$ em relação ao grupo controle com $p<0,001$. Não houve diferença significativa entre o grupo crioterapia e o grupo calor local. Considerações finais: conclui-se que os recursos térmicos associados ao alongamento são efetivos no pré-alongamento, sendo possível que o usuário escolha o melhor recurso.

Palavras-chave: Maleabilidade; Adolescente; Hipertermia Induzida; Crioterapia.

\section{ABSTRACT}

Objective: evaluate the effectiveness of two thermal resources in stretching hamstrings in adolescents. Method: non-randomized controlled clinical study with 45 male adolescents aged 13 and 16 years. Individuals with musculoskeletal lesion in the lower limbs, sensitivity deficit or physical activity practitioner that allowed gain of amplitude of movement were excluded from the study. The sample was divided in three groups, being cryotherapy, local heat and control. The groups were evaluated on the same day, at different times. With the aid of a goniometer, used before and after the intervention, a hip flexion was requested along with a knee extension and ankle dorsiflexion to the maximum of possible amplitude. After the first measurement, the interventions were performed with gel bags for 20 minutes, with ice at temperature below $0^{\circ} \mathrm{C}$ in group 1, and heat in group 2, whose temperature was on average $43^{\circ} \mathrm{C}$. The temperatures were controlled with the aid of a thermometer. After the intervention, a new measure was performed. In the control group, only two measurements were performed with the goniometer at intervals of 20 minutes. The mean and standard deviation were used for the descriptive statistics. For the 
comparison between groups, one-way variance analysis with Tukey post-hoc was used. For the analysis within each group, the paired t-test was used. Results: the two experimental groups (cryotherapy and local heat) effectively increased amplitude of movement in relation to the control group with $p<0.001$. There was no statistically difference between the cryotherapy group and the local heat group. Closing remarks: it is concluded that the thermal resources associated with stretching are effective in pre-stretching, and it is possible for the user to choose the best resource.

Keywords: Pliability; Adolescent; Hyperthermia Induced; Cryotherapy.

\section{INTRODUCÃO}

O número de praticantes de atividade física vem aumentando e, consequentemente, um alto índice de lesões musculares também passaram a ocorrer no cotidiano destes praticantes. As lesões podem ocorrer em qualquer indivíduo que se submeta à prática de exercícios físicos. ${ }^{1}$ Sendo assim, torna-se necessário estabelecer formas de prevenção, educação e reabilitação, tendo em vista que atividades físicas estão relacionadas com benefícios a curto e longo prazo para a saúde..$^{2,3}$

O alongamento, como prática de prevenção de lesões musculares, tem sido encorajado a fim de evitar o espasmo muscular e a dor isquêmica. Isto porque a mobilidade adequada dos tecidos moles e articulações é também um fator importante na prevenção de lesões. ${ }^{4}$ Exercícios de flexibilidade causam um aumento no comprimento da unidade músculo tendínea. Todavia, as propriedades viscosas dos tecidos fazem com que este aumento não seja imediatamente reversível. ${ }^{5} \mathrm{~A}$ flexibilidade muscular, definida como a capacidade de um músculo alongar-se, é indispensável em programas de prevenção e reabilitação, pois a mobilidade dos tecidos moles é fundamental para a execução das tarefas cotidianas. ${ }^{6}$ Nesse sentido, alongamento é o termo utilizado para as manobras terapêuticas elaboradas para aumentar a amplitude de movimento das estruturas encurtadas. ${ }^{7}$

Nesse sentido, o alongamento é capaz de promover aumento na extensibilidade muscular, tendo ação nas fibras musculares e tecido conjuntivo que as envolvem. ${ }^{8}$ Sua fisiologia envolve a atividade reflexa do músculo e o comportamento elástico da unidade músculo tendínea. Esta atividade reflexa é mediada por receptores sensíveis às variações de comprimento e tensão, chamados Fusos Musculares e Órgãos Tendinosos de Golgi (OTG). Os fusos musculares ajustam-se constantemente ao comprimento do músculo e são estimulados pelo aumento do comprimento deste. Ainda, promovem, por via reflexa, a contração muscular de maior ou menor intensidade, com duração variável. ${ }^{9}$

A utilização de recursos terapêuticos que atuem no componente neural pode diminuir a ativação reflexa do músculo, aumentando assim a tolerância ao alongamento e, com isto, a flexibilidade. Nesse sentido, a utilização da crioterapia é recomendada por atuar diretamente no fuso muscular e no OTG, reduzindo a velocidade de condução nervosa e na descarga fusal, diminuindo o estímulo de tensão muscular e a sensação dolorosa. Porém, há controvérsias em relação ao seu uso constante. Estudo de Faria e Queiroz ${ }^{10}$ demonstrou um aumento da rigidez tecidual e consequente redução da elasticidade dos tecidos à longo prazo, o que poderia limitar a flexibilidade. Assim, recursos que agissem aumentando a elasticidade tecidual, aquecendo o músculo, seriam mais eficientes na redução da resistência. ${ }^{10,11}$

A utilização prévia do calor no alongamento pode proporcionar melhor flexibilidade com o aumento do metabolismo do tecido e redução do desconforto. Assim, com a maior extensibilidade dos tecidos, ocorre um melhor relaxamento muscular, com reduzida sensibilidade do fuso ao alongamento, o que facilitará a ação do OTG, proporcionando maior facilidade na execução do movimento. ${ }^{11,12}$

Outro fator que altera a flexibilidade é a puberdade, que pode ser entendida como o processo de amadurecimento biológico e ocorre aproximadamente entre nove e quinze anos de idade, com o desenvolvimento da capacidade reprodutora. ${ }^{13} \mathrm{O}$ adolescente passa por um abrupto crescimento longitudinal recorrente das alterações hormonais, podendo apresentar um crescimento ósseo mais acelerado do que o crescimento dos tendões e músculos, o que acaba repercutindo na diminuição da flexibilidade, a qual fica mais propensa a lesões. ${ }^{14,15}$ Sendo assim, o objetivo deste estudo foi avaliar a eficácia de dois recursos térmicos no alongamento de isquiotibiais em adolescentes.

\section{MÉTODO}

O presente estudo, caracterizado como ensaio clínico controlado e não randomizado, contou com 45 adolescentes do sexo masculino. Os critérios de inclusão foram: ser voluntário e estar na faixa etária entre 13 e 16 anos. Os critérios de exclusão adotados foram: indivíduos com lesões musculoesqueléticas em membros inferiores, com déficit de sensibilidade, ou que realizassem atividade física que possibilitasse o ganho de amplitude de movimento nos membros inferiores. A direção da escola assinou o Termo de Autorização Institucional, assim como os meninos voluntários, e seus responsáveis, consentiram com o Termo Livre e Esclarecido (TCLE). Durante o protocolo de avaliação, não houve registro de perdas amostrais. Este estudo foi aprovado pelo Comitê de Ética e Pesquisa sob protocolo de número 212/2010.

Inicialmente, a amostra foi dividida em três grupos (Crioterapia, Calor local e Controle) com 15 meninos cada. Foi mensurada a amplitude de movimento da musculatura dos isquiotibiais em decúbito dorsal. Foi solicitado ao avaliado que realizasse uma flexão de quadril juntamente com uma extensão de joelho e dorsiflexão de tornozelo até o máximo de amplitude possível, caracterizando o alongamento estático, seguindo protocolo de Marques..$^{18} \mathrm{O}$ ângulo foi medido através de um goniômetro universal (FisioStore) com braço fixo na linha média axilar do tronco e braço móvel, paralelo e sobre a superfície lateral da coxa, em direção ao côndilo lateral do fêmur.

Para a aplicação dos recursos térmicos, os adolescentes permaneceram em decúbito dorsal. A crioterapia foi realizada através da aplicação de bolsas de gel BC0134 Quente-Fria Mercur diretamente sobre o ven- 
tre dos músculos isquiotibiais em temperatura abaixo de $0^{\circ} \mathrm{C}$ no primeiro grupo. Para a realização do calor local (grupo 2) as bolsas de gel foram mantidas a uma temperatura próxima à $43^{\circ} \mathrm{C}$, e aplicadas diretamente sobre o ventre muscular. As aplicações de frio e calor foram realizadas por 20 minutos conforme o protocolo. ${ }^{10,19}$ As temperaturas foram controladas com o auxílio de um termômetro de mercúrio. Após a intervenção, foi novamente mensurada a amplitude de movimento da musculatura posterior da coxa. No grupo controle foram realizadas apenas duas medidas com o goniômetro, sendo que entre a primeira e a segunda medida foi respeitado o intervalo de 20 minutos.

Para a estatística descritiva foram utilizadas a média, o desvio padrão, o erro padrão da média, além dos valores mínimos e máximos. Para a comparação entre os grupos foi utilizada a análise de variância de uma via (One-Way ANOVA) com post-hoc de Tukey. Já, para a análise dentro de cada grupo, foi utilizado o teste $t$ pareado. O nível de significância adotado foi de $5 \%$. Os dados foram analisados no programa estatístico SPSS for windows 20.0.

\section{RESULTADOS}

A amostra contou com 45 voluntários divididos em 3 grupos de 15 indivíduos. As médias de idade dos grupos crioterapia, calor local e controle foram 14,40 $\pm 0,73$ anos, $14,46 \pm 0,91$ anos e 14,13 $\pm 0,74$ anos, respectivamente.

Ao serem analisados os valores médios dos diferentes grupos antes e após a aplicação da intervenção (tabela 1), foi identificado, nos três grupos, aumento dos valores de ADM. O grupo controle, mesmo sem ser submetido à intervenção apresentou um aumento médio de $5,47^{\circ} \mathrm{C}$ passando de $56,80 \pm 9,79^{\circ} \mathrm{C}$ para $62,27 \pm 6,88^{\circ} \mathrm{C}$. Já, o grupo submetido à crioterapia apresentou aumento médio de $9,06^{\circ} \mathrm{C}$ passando de $68,67 \pm 7,43^{\circ} \mathrm{C}$ para $77,73 \pm 8,84^{\circ} \mathrm{C}$. Enquanto que o grupo submetido ao calor local apresentou aumento médio de $12,26^{\circ} \mathrm{C}$, passando de $73,73 \pm 13,58^{\circ} \mathrm{C}$ para $61,47 \pm 10,70^{\circ} \mathrm{C}$.

Conforme apresentado na tabela 2, o aumento apresentado nos valores de ADM antes e após a intervenção foram estatisticamente significativos nos três grupos (Grupo Controle: $p=0,000$; Grupo Crioterapia: $p=0,006$; Grupo Calor local: $p=0,000)$. Na comparação entre os grupos, a avaliação da ADM antes $(p=0,005)$ e após intervenção $(p=0,000)$ foram significativas $(p<0,05)$. Quanto à localização das diferenças, antes da aplicação da intervenção já existia diferenças significativas entre o grupo submetido à crioterapia e o grupo controle $(p=0,004)$. Após a intervenção, foi mantida a diferença entre o grupo submetido à crioterapia e o grupo controle $(p=0,000)$, passando a ocorrer diferenças significativas entre o grupo submetido ao calor local e o grupo controle $(p=0,010)$.

\section{DISCUSSÃO}

De acordo com os resultados, observa-se que os três grupos aumentaram efetivamente a amplitude de movimento articular dos músculos isquiotibiais. Todavia, aumentos mais expressivos foram apresentados pelos grupos crioterapia e calor local.

Ao analisar o ganho de amplitude de movimento do grupo submetido à crioterapia, verificou-se que o resfriamento muscular é um método eficaz. Seus efeitos fisiológicos são capazes de diminuir a velocidade dos nociceptores e os espasmos musculares. Segundo Brasileiro, Faria e Queiroz, ${ }^{10}$ a crioterapia age no fuso muscular, causando diminuição da velocidade de condução nervosa que reduz a descarga fusal, facilitando o estímulo de tensão muscular com menor disparo dos fusos. Para além disso, o frio causa uma vasoconstrição produzida pelas fibras simpáticas e por ação direta sobre os vasos. Desta forma, uma vez reduzida estas sensações, maior seria a tolerância do voluntário à manobra, permitindo um alongamento mais efetivo devido à diminuição da limitação álgica que precede à limitação tecidual. ${ }^{11,20}$

Em estudo de Brasileiro et al., ${ }^{10}$ foi demonstrado que o comportamento da crioterapia, em 40 adultos jovens, de ambos os sexos, com média de idade de $21,5 \pm 3,1$ anos, na flexibilidade dos músculos isquiotibiais, foi significativo $(p<0,01)$ quando comparado à grupos de calor local e de alongamento por contração-

Tabela 1 - Descrição dos valores de amplitude de movimento de alongamento de isquiotibiais antes e após as diferentes intervenções.

\begin{tabular}{|c|c|c|c|c|c|c|}
\hline Grupo & Intervenção & Mín. & Máx. & Méd. & DP & EPM \\
\hline \multirow[t]{2}{*}{ Controle } & ADM Pré & $40^{\circ} \mathrm{C}$ & $70^{\circ} \mathrm{C}$ & $56,80^{\circ} \mathrm{C}$ & $9,79^{\circ} \mathrm{C}$ & $2,52^{\circ} \mathrm{C}$ \\
\hline & ADM Pós & $50^{\circ} \mathrm{C}$ & $70^{\circ} \mathrm{C}$ & $62,27^{\circ} \mathrm{C}$ & $6,88^{\circ} \mathrm{C}$ & $1,77^{\circ} \mathrm{C}$ \\
\hline \multirow[t]{2}{*}{ Crioterapia } & ADM Pré & $56^{\circ} \mathrm{C}$ & $82^{\circ} \mathrm{C}$ & $68,67^{\circ} \mathrm{C}$ & $7,43^{\circ} \mathrm{C}$ & $1,91^{\circ} \mathrm{C}$ \\
\hline & ADM Pós & $64^{\circ} \mathrm{C}$ & $98^{\circ} \mathrm{C}$ & $77,73^{\circ} \mathrm{C}$ & $8,84^{\circ} \mathrm{C}$ & $2,28^{\circ} \mathrm{C}$ \\
\hline \multirow[t]{2}{*}{ Calor local } & ADM Pré & $50^{\circ} \mathrm{C}$ & $90^{\circ} \mathrm{C}$ & $61,47^{\circ} \mathrm{C}$ & $10,70^{\circ} \mathrm{C}$ & $2,76^{\circ} \mathrm{C}$ \\
\hline & ADM Pós & $60^{\circ} \mathrm{C}$ & $110^{\circ} \mathrm{C}$ & $73,73^{\circ} \mathrm{C}$ & $13,58^{\circ} \mathrm{C}$ & $3,50^{\circ} \mathrm{C}$ \\
\hline
\end{tabular}

Min. = mínimo; Máx. = máximo; Méd. = média; $A D M=$ amplitude de movimento; $D P=$ Desvio-padrão; E.P.M. = Erro Padrão da Média.

Tabela 2 - Comparação dentro de cada grupo dos valores médios de ADM.

\begin{tabular}{lccc}
\hline Grupo & ADM Pré Média \pm DP & ADM Pós Média \pm DP & S \\
\hline Controle & $56,80 \pm 9,79^{\circ}$ & $62,27 \pm 6,88^{\circ}$ & $-4,576$ \\
Crioterapia & $68,67 \pm 7,43^{\circ}$ & $77,73 \pm 8,84^{\circ}$ & $-3,261$ \\
Calor local & $61,47 \pm 10,70^{\circ}$ & $73,73 \pm 13,58^{\circ}$ & -000 \\
\hline
\end{tabular}

$A D M=$ Amplitude de Movimento; $D P=$ Desvio-padrão. 
-relaxamento. Porém, como efeito adverso, Knight ${ }^{11}$ afirma que o tecido conjuntivo aumenta sua rigidez e sua extensibilidade diminui conforme a temperatura declina. Já Mortari, Mânica e Pimentel, ${ }^{21}$ ao analisarem a influência da crioterapia na flexibilidade e na força muscular da musculatura flexora e extensora do joelho, obtiveram como resultado a redução da força concêntrica imediatamente após a aplicação da crioterapia. Herbert e Noronha ${ }^{4}$ demostraram que, após 30 minutos de resfriamento, ocorre aumento de força isométrica máxima imediatamente após a aplicação de frio, porém ao final do tratamento há um declínio na força muscular, sendo um fator negativo da aplicação de crioterapia.

Quanto aos resultados do calor local, diferentes estudos $^{22-24}$ tem demonstrado que o uso da hipertermia é eficaz na associação com o alongamento, pois o calor diminui o desconforto durante o alongamento pois aumenta a extensibilidade das fibras de colágeno, favorecendo o relaxamento das propriedades mecânicas do músculo com redução da sensibilidade do fuso muscular. Brasileiro et al. ${ }^{10}$ sugerem que a elevação da temperatura entre 3 a $4^{\circ} \mathrm{C}$, aumenta significativamente a extensibilidade dos tecidos e, com isto, é capaz de melhorar a flexibilidade. O aumento da temperatura central aumenta a produção de energia pela aceleração das redes oxidativas. Ainda, como efeito direto, ocorre um aumento na liberação de oxigênio da hemoglobina e mioglobina, aumento da taxa de reações metabólicas e tempo de condução nervosa, chegando mais fluxo de sangue no local e aumentando o consumo de oxigênio basal. Ao comparar os efeitos da hipertermia e hipotermia, Minato et al. ${ }^{26}$ demonstraram melhores resultados a partir da utilização do calor, onde foi identificado maior relaxamento muscular sem possíveis restrições. Os autores ainda destacam que a musculatura posterior tensa de um atleta corredor gera comprimento de passada limitado, o que faz com que ele dê mais passos numa determinada distância comparando ao número de passos se tivesse mais flexibilidade. ${ }^{26}$

Estudos referentes à associação de recursos térmicos ao alongamento em adolescentes são importantes devido ao grande crescimento longitudinal durante essa fase da vida. Nesse período, ocorre o fenômeno da puberdade, que tem como principal sinal o estirão de crescimento, epífises abertas, aumento da tração sobre os locais de inserção muscular, a rápida aquisição de conteúdo mineral ósseo e o processo de maturação esquelética. Os ossos crescem numa velocidade maior do que os tendões e músculos, supondo que a flexibilidade diminua com o aumento da idade e que isto possa contribuir para que possíveis problemas ortopédicos se instalem nessa faixa etária.

O crescimento físico é caracterizado pela somatória de fenômenos celulares, biológicos, bioquímicos e morfológicos, que se interagem através de um plano predeterminado geneticamente e influenciado pelo meio ambiente. Entre os aspectos ambientais, recebe destaque a nutrição que, conjugada a fatores hormonais e genéticos, promove a proliferação da cartilagem de crescimento e o alongamento linear dos ossos. ${ }^{27}$

Como limitação do estudo é possível sugerir o não controle da maturação biológica, o que pode ter interferido nos resultados do mesmo.

\section{CONSIDERAÇÕES FINAIS}

Dentro das condições experimentais propostas, sugere-se que recursos térmicos podem auxiliar a amplitude de movimento articular, o que juntamente ao alongamento podem auxiliar a flexibilidade dos músculos avaliados. Todavia, não há predominância de um recurso ser mais efetivo do que o outro. A decisão da melhor intervenção deve ser auxiliada pelo profissional, seguindo as melhores condutas fisioterapêuticas.

\section{REFERÊNCIAS}

1. Clebis NK, Natali MRM. Lesões musculares provocadas por exercícios excêntricos. Rev Bras Ciên Mov. 2001; 3(9):47-53.

2. Halall PC. Prática de atividade física em adolescentes brasileiros. Ciênc Saúde Coletiva 2010;15(2):3035-42. doi: 10.1590/S1413-81232010000800008

3. Cruz CL; Gotardo C; Jorge S. Influência da crioterapia e do calor superficial na espasticidade - Relato de caso. Arq Ciênc Saúde Unipar 2003;7(3):253-57.

4. Herbert RD, de Noronha M, Kamper SJ. Stretching to prevent or reduce muscle soreness after exercise. Cochrane Database Syst Rev 2011;6(7):CD004577. doi: 10.1002/14651858. CD004577.pub3.

5. Viveiros L, Polito MD, Simão R, Farinatti P. Respostas agudas imediatas e tardias na flexibilidade na extensão do ombro em relação ao número de séries e duração do alongamento. Rev Bras Med Esporte 2004;10(6):459-63. doi: 10.1590/S151786922004000600002

6. Bonvincine C, Gonçalves C, Batigália F. Comparação do ganho de flexibilidade isquiotibial com diferentes técnicas de alongamento passivo. Acta Fisiatr 2005;12(2):43-7.

7. Kisner C, Colby LA. Exercícios terapêuticos, fundamentos e técnicas. São Paulo: Manole. 1998.

8. Silva NL, Farinatti PTV. Influence of counter-resistance training variables on elderly muscular strength: a systematic review with emphasis on dose/response relationships. Rev Bras Med Esporte 2007;13(1):51-7

9. Cabral CS, Goldberg TB, Teixeira AS. O exercício físico potencializa ou compromete o crescimento longitudinal de crianças e adolescentes? Mito ou verdade? Rev Bras Med Esporte 2004;10(6):520-24. doi: 10.1590/S151786922004000600009

10. Brasileiro JS, Faria AF, Queiroz LL. Influência do resfriamento e do aquecimento local na flexibilidade dos músculos isquiotibiais. Rev Bras Fisioter 2007;11(1):57-61. doi: 10.1590/S1413-35552007000100010

11. Knight K. Crioterapia no tratamento das lesões esportivas. São Paulo: Manole. 2000.

12. Voigt L, Vale RGS, Abdala DW, Freitas WZ, Novaes JS, Dantas EHM. Efeitos de uma repetição de dez segundos de estímulo do método estático para o desenvolvimento da flexibilidade de homens adultos jovens. Fit Perf J 2007;6(6):352-6. doi: 10.3900/fpj.6.6.352.p

13. Zeferino $A M B$, Barros Filho $A A$, Bettiol $H$, Barbieri MA. Acompanhamento do crescimento. JPediatr 2003;17(3):S23-S32. doi: 10.1590/S0021-75572003000700004

14. Mikkelsson LO, Nupponen $\mathrm{H}$, Kaprio J, Kautiainen $\mathrm{H}$, Mikkelsson M, Kujala UM. Adolescent flexibility, endurance strength, and physical activity as predictors of adult tension neck, low back pain, and knee injury: a 25 year follow up. Br J Sports Med 2006;40(2):107-13. doi: 10.1136/ 
bjsm.2004.017350

15. Silva RJS. Características de crescimento, composição corporal e desempenho físico relacionado à saúde em crianças e adolescentes de 07 a 14 anos da região do cotinguiba (SE). 2002. Dissertação (Mestrado). Universidade Federal de Santa Catarina, 2002.

16. Castilho RF, Silva AS. Doença de Osgood Schlatter - Revisão Bibliográfica e Proposta de tratamento. Revista Inspirar 2009;1(2):24-35

17. Ribeiro MC. Tratamento fisioterapêutico na patologia de Osgood Schlatter através do uso de laserterapia e cinesioterapia: estudo de caso. Trabalho de Conclusão de Curso- Cascavel, 2003.

18. Marques AP. Manual de Goniometria. São Paulo: Manole. 1997. p. 111

19. Camara FC, De Lima GM, Fantini G, Hadad PJ. Efeitos da utilização da crioterapia e do calor superficial na espasticidade de pacientes com lesão medular. Revista da Unorp 2005;4(12):7-23.

20. Hooper J, Salisbury L, Shuback B. A comparission of a self stretch incorporating proprioceptive neuromuscular facilitation components and a therapist applied PNF technique on hamstrings flexibility. Physiotherapy 2004;90(3):151-7. doi: 10.1016/j.physio.2004.02.009

21. Mortari DM, Mânica AP, Pimentel GL. Efeitos da crioterapia e facilitação neuromuscular proprioceptiva sobre a força muscular nas musculaturas flexora e extensora de joelho. Fisioter Pesqui 2009;16(4):329-34. doi: 10.1590/S180929502009000400008

22. Knight CA, Rutledge CR, Cox ME, Acosta M, Hall SJ. Effect of superficial heat, deep heat, and active exercise warm-up on the extensibility of plantar flexors. Phys Ther 2001;81(6):1206-14.

23. Bishop D. Warm up I: Potential mechanisms and the effects of passive warm up on the exercise performance. Sports Med 2003;33(6):439-54.

24. Rosa AC, Montandon I. Efeitos do aquecimento sobre a amplitude de movimento: uma revisão crítica. Rev Bras Ci e Mov 2006;14(1):109-16.

25. Signori LU, Voloski FRS, Kerkhoff AC, Brignoni L, Plentz RDM. Efeito de agentes térmicos aplicados previamente a um programa de alongamentos na flexibilidade dos músculos isquiotibiais encurtados Rev Bras Med Esporte 2008;14(4):32831. doi: 10.1590/S1517-86922008000400001

26. Minatto G, Ribeiro RR, Achour Junior A, Santos KD. Idade, maturação sexual, variáveis antropométricas e composição corporal: influências na flexibilidade. Rev Bras Cineantropom Desempenho Humano 2010;12(3):151-58.

27. Silva SA, Oliveira DJ, Jaques MJN, Araújo RC. Efeito da crioterapia e termoterapia associados ao alongamento estático na flexibilidade dos músculos isquiotibiais, Motricidade 2010;6(4):55-62.

Como citar: DE CASTRO, Nathália Fernandez; SILVA, Marcelo Faria; GARLIPP, Daniel Carlos. Comparação dos resultados de aplicação de calor local e crioterapia no alongamento de ísquiostibiais em adolescentes. Cinergis, Santa Cruz do Sul, v. 18, n. 2, mar. 2017. ISSN 2177-4005. Disponível em: <https://online.unisc.br/seer/index.php/cinergis/article/view/8791>. Acesso em: 29 mar. 2017. doi:http://dx.doi.org/10.17058/cinergis. v18i2.8791. 